\title{
State Estimation Using Augmented Blocked Matrices
}

\author{
Fernando L. Alvarado, Senior Member \\ The University of Wisconsin - Madison, Wisconsin
}

\section{ABSTRACT}

Blocked structures of the augmented matrix of power system state estimation are studied and it is shown that there are advantages in blocking according to a nodal formulation. The topological and computational properties of three blocking alternatives are studied and their numerical condition numbers compared. Recommendations are given for developing software for the proposed nodal blocking formulation. Keywords: Sparsity, State Estimation, Sparse Matrices, Matrix Conditioning.

\section{INTRODUCTION}

The static state estimation problem in power systems can be defined as the solution to the following mathematical problem:

Given:

- an unknown vector of $n$ state variables $x$

- a known vector of $m$ measurements $z$

- a known function $h: R^{n} \rightarrow R^{m}$

- a vector of $m$ residuals $r$ defined as: $r=z-h(x)$

- a known function $\mathrm{g}: \mathrm{R}^{\mathrm{n}} \rightarrow \mathrm{R}^{\mathrm{p}}$

- a vector of p equality constraints on $x$ : $g(x)=0$

Find:

- a value for $\mathrm{x}$ that minimizes $\mathrm{r}^{\mathrm{t}} \mathrm{W} r$, the weighted sum of the squares of the residuals. $W$ is a (usually diagonal) $\mathrm{m}$ by $\mathrm{m}$ matrix.

All methods considered rely equally on iterative computations. Therefore only the linearized version of the state estimation problem is considered here. The linearized state estimation problem is:

Given:

- an unknown vector of $\mathbf{n}$ state variables $\mathbf{x}$

- a known vector of $m$ measurements $z$

- a known $m$ by $n$ matrix $H$

- a vector of $m$ residuals $r$ defined as: $r=z-H x$

- a known $\mathrm{p}$ by $\mathrm{n}$ matrix $\mathrm{G}$

- a vector of $p$ equality constraints on $x: G x=g$

Find:

- a value for $x$ that minimizes $r^{t} W r$.

The solution to this problem requires methods that are both computationally efficient and numerically robust.

90 Wh 241-0 PWhS A paper recommended and approved by the IEE Power System Engineering Comittee of the IEEE Power Engineering Society for presentation at the IEED/PES 1990 Winter Heeting, Atlanta, Georria, February 4-8, 1990. Manuscript submitted Ausust 28, 1989: made available for printing January 18, 1990.

\author{
William F. Tinney, Fellow \\ Consultant - Portland, Oregon
}

Known methods for improving numerical robustness tend to reduce speed. The method introduced here improves robustness of the most widely used formulation of state estimation with very little, if any, sacrifice in speed. The method is based on a particular block structure and the use of block arithmetic in matrix operations. Block matrix methods and their importance in power system computations were described in [3]. The block method used in this paper is similar to the method used in [1] for contingency ranking.

\section{EXISTING STATE ESTIMATION METHODS}

In this section the main known methods to solve the state estimation problem are reviewed. The small 8 node example of Figure 1 is used to illustrate these methods.

The $\mathrm{H}$ matrix is divided into two parts, a part corresponding to branch measurements and a part corresponding to nodal injection measurements. The topology of $\mathrm{G}$ and $\mathrm{H}$ for this example is:

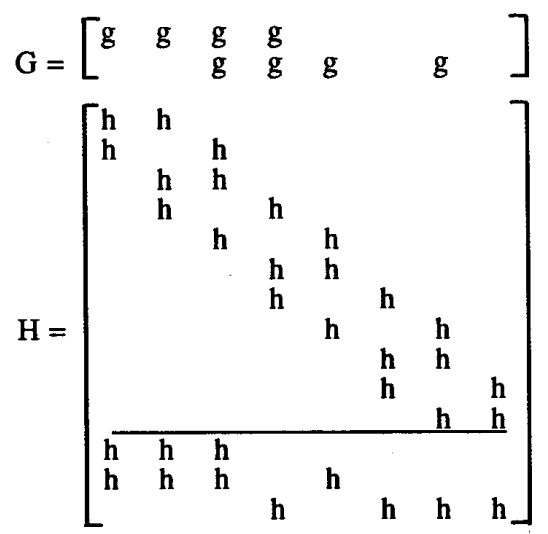

Following are the main formulations and solution methods for the state estimation problem:

Traditional formulation without equality constraints

$$
\text { Minimize } J=\frac{1}{2}[z-H x]^{t} W[z-H x]
$$

This problem is solved from:

$$
\left[\mathrm{H}^{\mathrm{t}} \mathrm{WH}\right] \mathrm{x}=\mathrm{H}^{\mathrm{t}} \mathrm{W} z
$$

Approximate handling of equality constraints

$$
\text { Minimize } J=\frac{1}{2}[z-H x]^{t} W[z-H x]+\frac{1}{2}[g-G x]^{t} V[g-G x]
$$

$\mathrm{V}$ is a diagonal matrix of artificial weights. Large values of $\mathrm{V}$ make the equality constraints more rigid, but they deteriorate the numerical properties of the solution. This problem is solved from:

$$
\left\{\left[H^{t} W H\right]+\left[G^{t} V G\right]\right\} x=H^{t} W z+G^{t} V g
$$




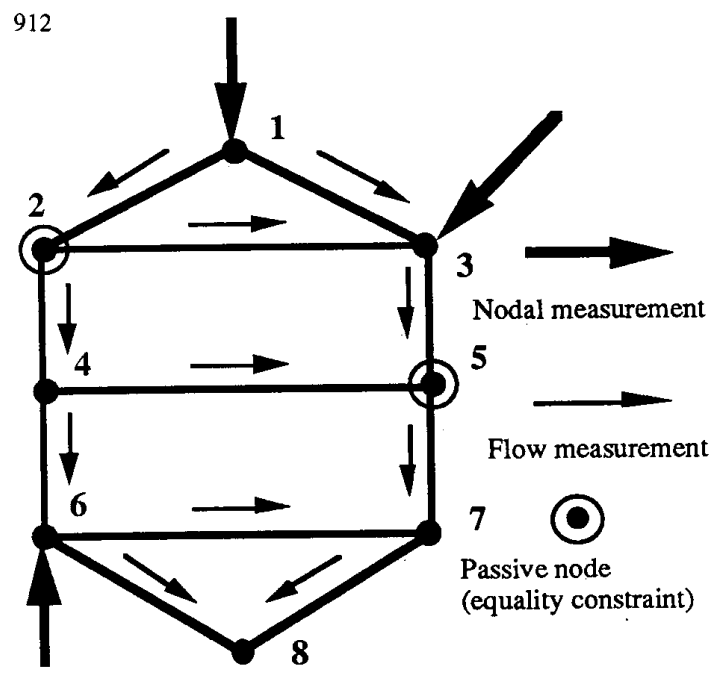

Figure 1: Sample system with 8 nodes, 11 branches, 11 branch measurements, 3 nodal injection measurements (nodes 1, 3 and 6 ) and 2 passive nodes (nodes 2 and 5).

\section{Exact enforcement of equality constraints [5]}

$$
\begin{gathered}
\text { Minimize } J=\frac{1}{2}[z-H x]^{t} W[z-H x] \\
\text { subject to } G x=g
\end{gathered}
$$

This problem is solved by explicit introduction of Lagrange multipliers $\lambda$ :

$$
\left[\begin{array}{cc}
\mathrm{H}^{\mathrm{t}} \mathrm{WH} & \mathrm{G}^{\mathrm{t}} \\
\mathrm{G} & 0
\end{array}\right]\left[\begin{array}{c}
\mathrm{x} \\
\lambda
\end{array}\right]=\left[\begin{array}{c}
\mathrm{H}^{\mathrm{t} W z} \\
\mathrm{~g}
\end{array}\right]
$$

\section{Augmented matrix (Hachtel) method, no constraints [15]}

$$
\begin{gathered}
\text { Minimize } J=r^{t} W r \\
\text { where } r=W^{-1} \mu=z-H x
\end{gathered}
$$

This problem is solved from the augmented equations:

$$
\left[\begin{array}{cc}
\mathrm{D} & \mathrm{H} \\
\mathrm{H}^{\mathrm{t}} & 0
\end{array}\right]\left[\begin{array}{l}
\mu \\
\mathrm{x}
\end{array}\right]=\left[\begin{array}{l}
\mathrm{z} \\
0
\end{array}\right]
$$

Where $D=W^{-1}$. The augmented method requires that the computations be properly organized. There are many possibilities for doing this. Which is best depends on the application. Because at the onset many of the diagonal elements in the augmented matrix are zero or very small, it is not possible to select diagonal pivots arbitrarily. There are three possibilities:

- Perform diagonal pivoting but defer the selection of an element as a pivot until its numeric value becomes sufficiently large.

- Perform off-diagonal pivoting, making the matrix unsymmetric.

- Allow for the automatic recognition of nonsingular 2 by 2 blocks which include a zero component along the diagonal. This approach was introduced by $[9,10]$. It is effective but does not realize all the benefits that can be attained in this type of problem, as this paper illustrates.

\section{Augmented matrix with constraints [12]}

A slight extension of the above method permits the introduction of equality constraints into the formulation:

$$
\begin{gathered}
\text { Minimize } \mathrm{J}=\mathrm{r}^{\mathrm{t}} \mathrm{W} \mathrm{r} \\
\text { Subject to } \mathrm{r}=\mathrm{W}^{-1} \mu=\mathrm{z}-\mathrm{Hx} \text { and } \mathrm{Gx}=\mathrm{g}
\end{gathered}
$$

This problem is solved from:

$$
\left[\begin{array}{ccc}
0 & 0 & G \\
0 & D & H \\
G^{t} & H^{t} & 0
\end{array}\right]\left[\begin{array}{l}
\lambda \\
\mu \\
x
\end{array}\right]=\left[\begin{array}{l}
g \\
z \\
0
\end{array}\right]
$$

The computational implications of using this formulation are described in the discussion and closure to $[14]$ and in [21].

\section{Pseudo-inverses method, no constraints $[6,13,16]$}

This method (also known as the method of Peters and Wilkinson) performs least squares errcr minimization on a transformation of the original problem. First all references to $\mathrm{W}$ are eliminated by re-defining $\mathrm{H}$ and $\mathrm{z}$ :

$$
\mathrm{A}=\mathrm{W}^{-1 / 2} \mathrm{H} \quad \mathrm{b}=\mathrm{W}^{-1 / 2} \mathrm{z}
$$

The problem becomes:

$$
\text { Minimize } \tilde{\mathbf{r}}^{\mathrm{t}} \tilde{\mathbf{r}} \text { where } \tilde{\mathbf{r}}=\mathrm{A} \mathrm{x}-\mathrm{b}
$$

The matrix $A$ continues to be an $m$ by $n$ matrix. It can be factored into factors $\mathrm{L}$ and $\mathrm{U}$, where $\mathrm{L}$ is an $\mathrm{m}$ by $\mathrm{n}$ matrix and $\mathrm{U}$ is a nonsingular $\mathrm{n}$ by $\mathrm{n}$ matrix. This factorization is not unique. In order to keep $U$ nonsingular renumbering of the rows and columns of $\mathrm{A}$ is usually required (off-diagonal pivoting). A most convenient factorization of $\mathrm{A}$ is to require that $\mathrm{L}$ be lower trapezoidal, and that $U$ be upper triangular:

$$
\begin{array}{ll} 
& A=L U \\
\text { Define: } & y=U x
\end{array}
$$

The original state estimation problem can be solved by first solving the following least squares error problem:

$$
\text { Minimize } \tilde{\mathbf{r}}^{\mathrm{t}} \tilde{\mathbf{r}}
$$

$$
\text { Subject to } \tilde{\mathbf{r}}=\mathrm{L} \text { y }-\mathrm{b}
$$

This transformed problem is solved for $\mathrm{y}$ from:

$$
\left[\mathrm{L}^{\mathrm{t}} \mathrm{L}\right] \mathrm{y}=\mathrm{L}^{\mathrm{t}} \mathrm{b}
$$

Solution of these equations requires the use of conventional sparsity-preserving factorization. The solution to the original problem is obtained by solving the upper triangular system of equations:

$$
\mathrm{U} \mathrm{x}=\mathrm{y}
$$

The numerical properties of the pseudo-inverse method can be shown to be better than those of the traditional method [16].

\section{Unconstrained Orthogonal Factorization [19,20,23]}

This method solves the minimization problem by first performing an orthogonal factorization of the A matrix. The A matrix is factored into the product of a rectangular orthogonal matrix $\mathrm{Q}$ and an upper triangular matrix $\mathrm{U}$. Solution to the estimation problem proceeds in two steps: 
- Let $y=Q^{t} b$

- Solve the triangular equations $U \mathrm{x}=\mathrm{y}$ for $\mathrm{x}$

The matrix $U$ has "second-neighbors" topology. As in the pseudo-inverses method, ordering of rows and columns of $\mathrm{A}$ is required to preserve sparsity. Constraints are treated as measurements with large weighting factors.

\section{Pseudo-inverses with equality constraints}

This method was introduced by Clements et al. [7]. The equality and measurement matrix equations are organized into a single partitioned matrix problem as:

$$
\left[\begin{array}{l}
G \\
A
\end{array}\right] x=\left[\begin{array}{l}
g \\
b
\end{array}\right]
$$

This method performs the same factorization as the method of pseudo-inverses. It yields a lower trapezoidal and a nonsingular upper triangular matrix for this combined set of equations, except that the renumbering of the rows of the matrix is confined within each group. That is, all rows of $\mathrm{G}$ are numbered ahead of all rows of A. The result is a lower trapezoidal matrix $L$ of dimension $m+p$ by $n$ and an upper triangular matrix $U$ of dimension $n$ by $n$. The lower trapezoidal matrix $L$ has the following structure:

$$
\left[\begin{array}{lc}
\mathrm{L}_{11} & 0 \\
\mathrm{~L}_{21} & \mathrm{~L}_{22}
\end{array}\right]
$$

The dimension of $L_{11}$ is $p$ by $p$. The dimension of $\mathrm{L}_{22}$ is $\mathrm{m}$ by ( $\left.\mathrm{n}-\mathrm{p}\right)$. The original problem can be solved by first solving an ordinary set of permuted equations based on $\mathrm{L}_{11}$ for an intermediate variable $\mathrm{w}$, then solving a permuted modified least squares problem for an intermediate variable $y$ (based on $\mathrm{L}_{2}$ ), and finally solving for the original variables of interest. The key steps are:

- Solve $L_{11} w=g$ for $w$ by forward substitution

- Solve $\mathrm{L}_{22}^{\mathrm{t}} \mathrm{L}_{22} \mathrm{y}=\mathrm{L}_{22}^{\mathrm{t}}$ b for y by sparse factorization

- Solve $U x=\left[\begin{array}{l}w \\ y\end{array}\right]$ for $x$ by back substitution

\section{THE BLOCKED FACTORS METHOD}

The computational burden of existing methods of state estimation is increased when nodal injection measurements are included because they create "secondneighbor" fill-in. Numerical difficulties are caused by the matrix products involved in the formulations and/or a wide range of magnitudes in the weighting factors.

The key idea of this paper is the observation that if the elements of the augmented matrix are grouped in blocks that correspond to nodes, the resulting blocked augmented matrix has the same topology as the familiar first-neighbor matrices of the power flow Jacobian and of Y-bus. The sparsity properties of these matrices are well understood and excellent methods for sparsity preservation exist. Both traditional ordering methods [17] and newer ordering algorithms which have some advantages over the traditional ordering schemes $[2,4]$ can be used.
The augmented Hachtel matrix for the example is:

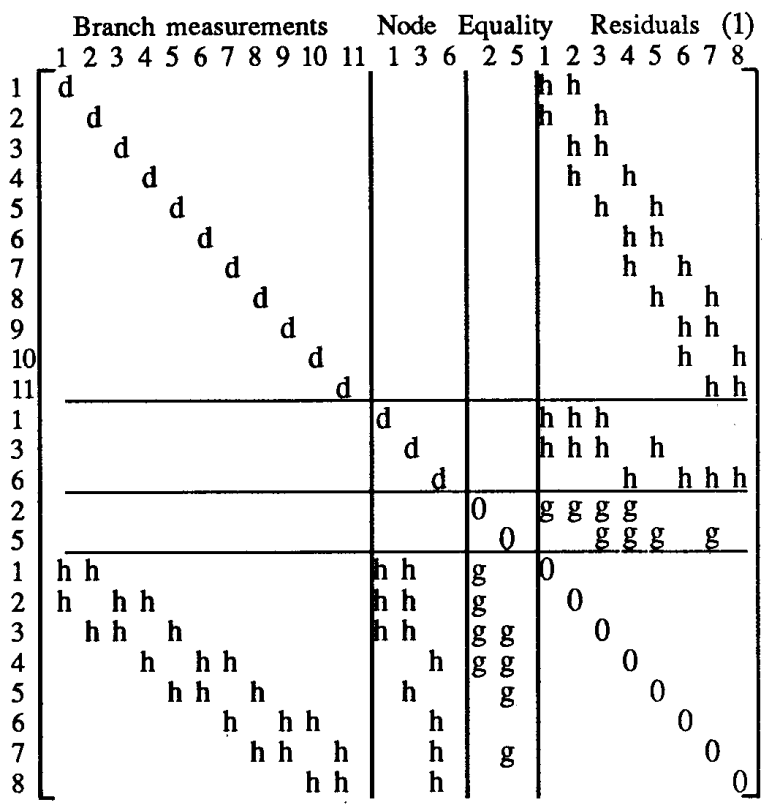

A partial factorization is performed to eliminate branch and nodal measurements. Equality constraint equations are retained. The result is a reduced matrix problem where the lower right hand corner fills in with a topology corresponding to first-neighbors for branch measurements and second-neighbors for nodal measurements. The result is:

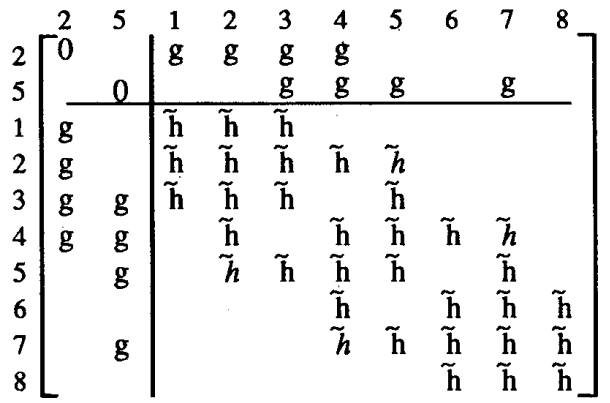

The $\tilde{\mathrm{h}}$ and $\tilde{h}$ entries refer to values that result from the incomplete factorization of the matrix, i.e., the elimination of branch and node measurements. Italicized entries $\tilde{h}$ refer to second-neighbor entries that result from elimination of nodal measurements. These equations can be written in "blocked" form by grouping terms that correspond to the same node. For example, node 2 is referred to in the upper rows of the matrix; it is also referred to in the lower rows of the matrix. These two rows can be grouped. Similarly, the two columns that refer to node 2 can be grouped. References to node 5 can also be grouped into blocks. The result is a blocked matrix: 


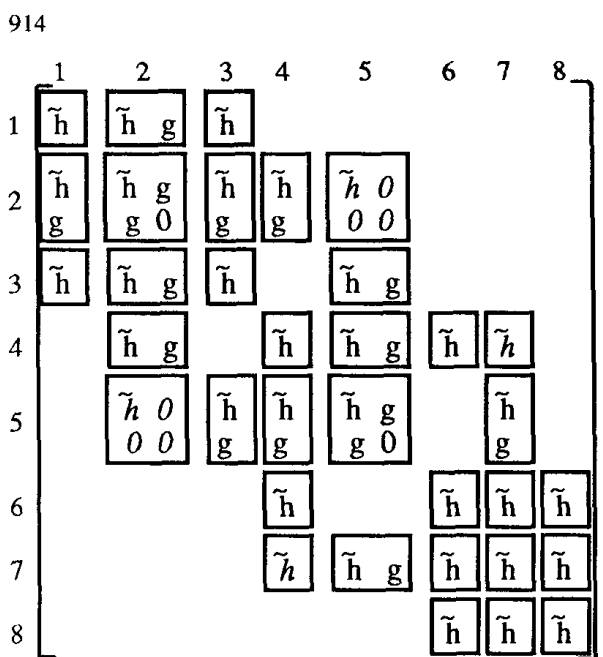

As an alternative, only the upper left hand portion containing branch measurements can be eliminated. The nodal measurements part of the matrix is not eliminated. Parts of the matrix corresponding to nodal measurements and equality constraints are blended into a single group. The proposed new method results in a matrix that has a firstneighbors topology pattern:

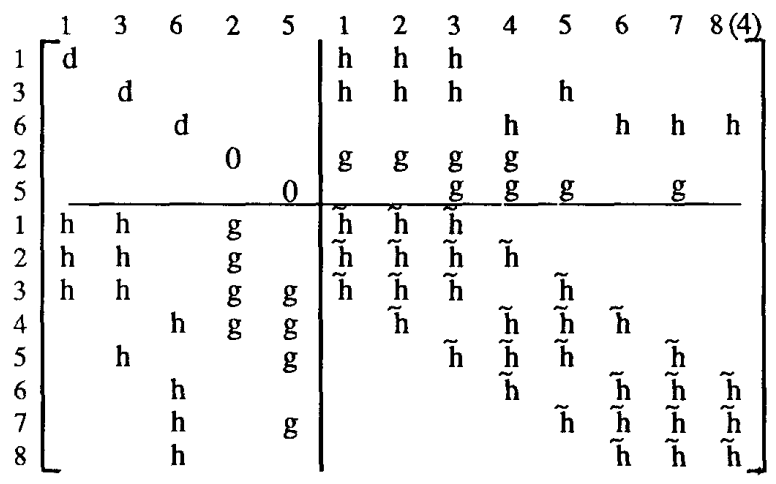

Matrix (4) can be blocked as follows:

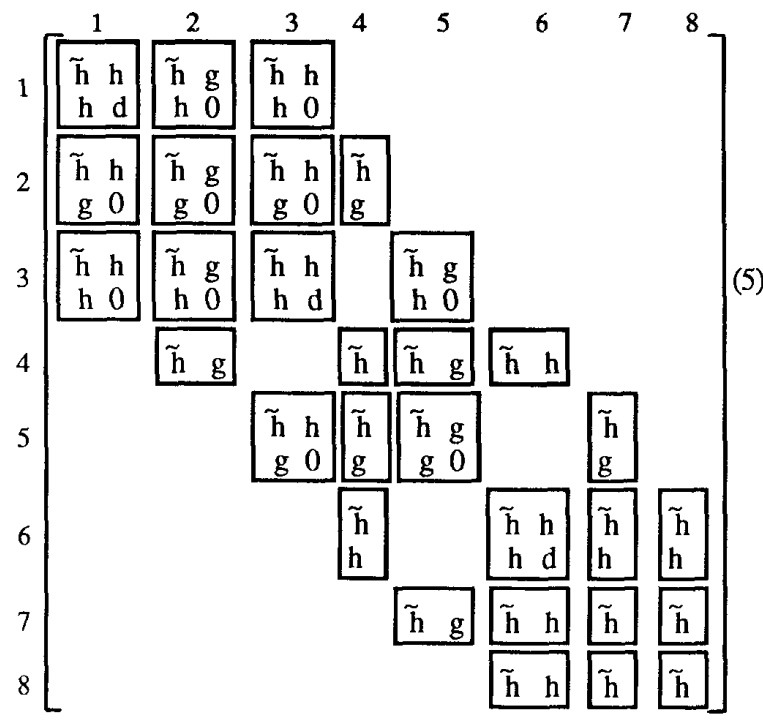

This matrix is solved using sparsity-preserving $U^{t} D U$ factorization, using block-arithmetic for all operations. This means, among other things, that diagonal blocks are inverted explicitly. Even though the blocks may have zeros on their diagonals, their inverses are well defined.

Although there is no second-neighbor fill-in for the blocked arrangement, the zero elements within the blocks have to be counted the same as the nonzero elements, and therefore may offset or actually increase the total number of nonzero floating point operations and storage requirements.

State estimation in power systems usually results in two equations for each branch measurement, one for $\mathrm{P}$ and one for $\mathrm{Q}$. There are also two variables to be estimated at each node, voltage magnitude and angle. Nodal measurements and constraints also come in pairs. Therefore, each of the "elements" $h$ and $g$ in the above matrices is actually a 2 by 2 matrix. Thus, the dimension of the blocks that are dealt with are 2 by 2,2 by 4,4 by 2 , or 4 by 4 . More detailed internal block topology is not illustrated here because it does not contribute to understanding of the method, but a production-grade implementation would require processing of all these block types.

It is often useful to compute certain elements of the residual co-variance matrix. The traditional formula for the residual co-variance matrix is:

$$
\mathrm{R}=\mathrm{W}^{-1}-\mathrm{H}\left(\mathrm{H}^{\mathrm{t}} \mathrm{WH}\right)^{-1} \mathrm{H}^{\mathrm{t}}
$$

Elements of $\left(\mathrm{H}^{\mathrm{t}} \mathrm{WH}\right)^{-1}$ can be computed directly from the augmented formulation in equation (5), and these values used in equation (6). Computation of post-estimate quantities is not directly affected by the blocking scheme. It appears, however, that other forms of blocking may be used to speed up these computations, but this has not yet been tried.

\section{EXAMPLES}

This section illustrates topological and numerical examples of the proposed blocking method for power systems ranging from 118 to 707 nodes. In all the comparisons the following is assumed:

- There is a branch measurement at every branch.

- There is a nodal injection measurement at one out of every three nodes. That is, there are injection measurements at nodes $1,4,7$, etc.

- One out of every three nodes is a passive node (an equality constraint). That is, the injections at nodes $2,5,8$, etc. are treated as equality constraints.

- The actual topology of the example systems is retained but their numerical values are replaced with a more consistent set that permits better comparisons of the numerical properties of different size networks.

All figures, operation counts and condition number calculations have been performed using the Sparse Matrix Manipulation System, a prototyping sparse matrix manipulation package [2].

\section{Sample Topology Diagrams}

In Figure 2 the topology diagram of the matrix that results from the conventional method is compared with that of the blocked matrix from [12] and with the topology of the 
blocked matrix proposed in this paper. These comparisons are illustrated for a 118 node system.

\section{Numerical Conditioning}

Numerical ill-conditioning has been associated with state estimation matrices. The degree of ill-conditioning of a matrix can be measured using the notion of "condition number," which is the norm of a matrix times the norm of its inverse. The norm used in this paper is the one-norm:

$$
\|A\|_{1}=\max _{j} \sum_{i}\left|a_{i j}\right|
$$

The condition number: $\kappa(\mathrm{A})=\|\mathrm{A}\|\left\|\mathrm{A}^{-1}\right\|$

For more information on matrix norms and condition numbers refer to [8]. The smallest possible condition number for a matrix is one. Small values (below 100) generally indicate good condition and few numerical difficulties. Large values (greater than 1000) indicate ill conditioning. Multiplying a matrix times itself squares its condition number. It is not hard to see, therefore, that methods that rely on the explicit computation of $\mathrm{H}^{\mathrm{t}} \mathrm{H}$ are likely to be ill-conditioned.

When ill-conditioning is a potential problem, certain measures can be taken to minimize its effects. These include QU factorization based on orthogonalization $[19,20]$, and the use of the method of pseudo-inverses, which avoids many (but not all) of the condition number problems.

A good way to avoid ill-conditioning is to avoid the operations that increase the condition number in the first place. The proposed method avoids computing the matrix products that lead to large condition numbers; therefore it never lets the condition number deteriorate.

\section{Tabular Comparisons}

Three methods are compared using three different matrices and two criteria. The methods compared are:

- The "traditional" method with equality constraints handled by using larger weights. The weights used are 10 times the unweighted values. (Note: typical values could actually be much greater).

- The augmented method, where blocking is used to deal with zeroes in the diagonal.

- The proposed method, where submatrix blocks are used not only to deal with zeroes but with all nodal equations.

Table 1: Comparison of computational requirements.

\begin{tabular}{|l|c|c|c|}
\hline Size & Traditional & Augmented & Proposed \\
\hline 118 & 8768 & 9656 & 6844 \\
\hline 352 & 19469 & 18882 & 15469 \\
\hline 707 & 67819 & 70345 & 67457 \\
\hline
\end{tabular}

Table 2: Comparison of condition numbers.

\begin{tabular}{|l||c|c|c|}
\hline Size & Traditional & Augmented & Proposed \\
\hline 118 & 8600 & 1390 & 227 \\
\hline 352 & 29470 & 1992 & 403 \\
\hline 707 & 28390 & 3712 & 466 \\
\hline
\end{tabular}

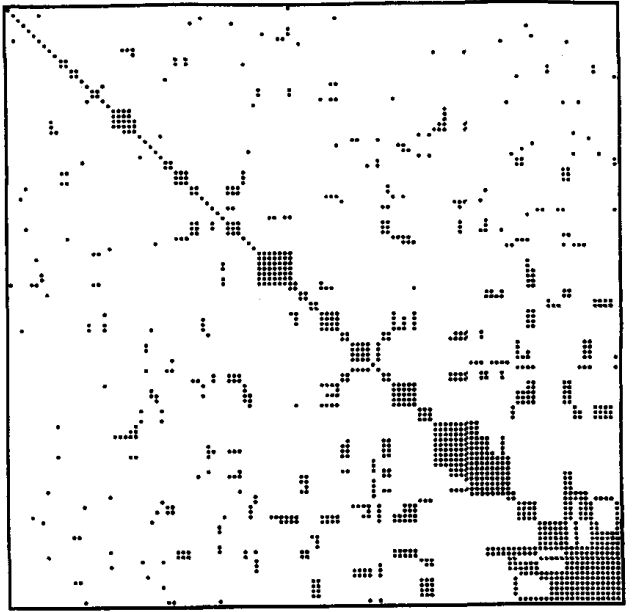

(a) The normal equations method. Dimension is 118 .

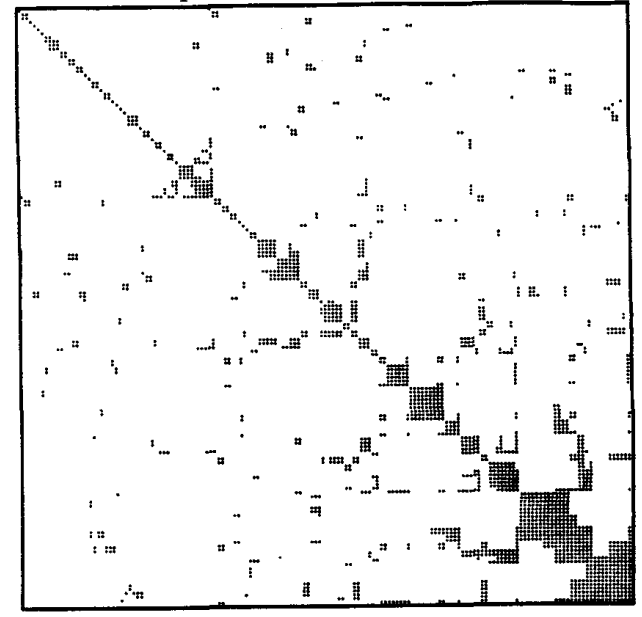

(b) Augmented method. Dimension is 118 blocks or 157 individual elements. Individual elements shown.

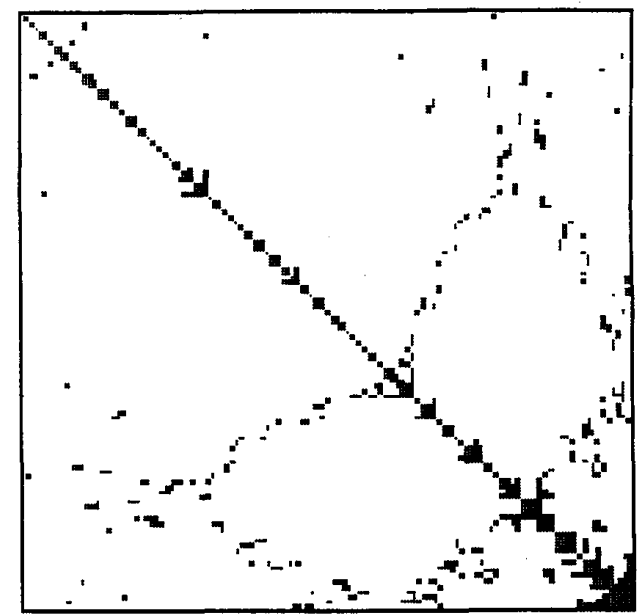

(c) The proposed method. Dimension is 118 blocks or 197 individual elements. Individual elements shown.

Figure 2: Comparison of topologies for 118 node system. Each dot represents an individual element within a block. 
The systems on which these comparisons are performed are all based upon actual power system topologies, ranging from 118 to 707 nodes. The comparison criteria are:

- Computational effort, measured as the total number of multiplications required for a complete $U^{t} D U$ factorization step. All multiplications are counted, including those needed to construct the matrix. No advantage is taken of symmetry or other computational shortcuts.

- Condition numbers are measured according to the onenorm of the matrix.

In an actual implementation, matrix symmetry normally would reduce the indicated operations counts by almost one half. Since this reduction would be approximately the same for each of the methods compared, the relative counts, which are what matter, would be unaffected. A more detailed comparison would also require an estimation of the computational effort of the sparsity logic for each method. In general, block operations reduce the relative cost of sparsity logic, compared to floating point arithmetic. The larger the blocks, the lower the ratio of logical to arithmetic operations.

The matrices tested had one third of all nodes designated as equality constraints. The benefits of the proposed method increase as the number of nodal measurements or constraints increases. However, the method proves advantageous even when a smaller number of nodal measurements is considered. The test for the 118 node system was repeated with $10 \%$ equality constraints instead of $33 \%$ and with weighting factors of 1000 instead of 10 . In this test the condition number was greater than $10^{6}$. This compares with 227 for the proposed method, which was unaffected. Additional extensive comparisons among all algorithms are described in [22]. These comparisons indicate that the condition number of the Givens method is superior, but that the proposed method compares well as far as number of operations.

Some investigators have contended that convergence is improved by using weighting factors instead of the Lagrange multipliers for equality constraints because the weighting factors "soften" the constraints. Any desired amount of "softness" in constraint enforcement can be obtained with the proposed method by adding suitable weighting factors to the normally zero diagonals of the exact equalities. Such added weighting factors will not worsen the condition number compared to the case of zero diagonals for exact equality constraint enforcement.

\section{CONCLUSIONS}

A new method of blocking the Hachtel formulation for power system state estimation is proposed. As indicated by its large reduction in the numerical condition number of three representative test problems, it should be able to overcome the usual causes of ill-conditioning of power system state estimation. As shown by comparisons of its computational requirements with those of other methods, there is no sacrifice in speed to achieve robustness.

The key idea is organization of the Hachtel formulation into a submatrix block structure that conforms to that of the incidence matrix of the network. This leads to the normal first-neighbor topological structure of the blocked matrix which is processed in the same sparsitypreserving order as the nodal admittance matrix. Explicit inversion of diagonal submatrix blocks overcomes former difficulties with small or zero diagonals and further enhances numerical stability.

Equality constraints are enforced exactly instead of as measurements with artificially large weighting factors. Another feature of the method is that it can accommodate a more realistic covariance matrix having nonzero off-diagonal weighting factors corresponding to correlations between the active and reactive power measurements at a bus. In fact, any covariance terms that fall within the submatrix blocks (in locations that are now zeroes) can be accommodated with no effect on computational burden.

This paper has not considered convergence issues in state estimation. Convergence of the proposed method should be nearly identical to that of the other state estimation methods when ill-conditioning does not play a significant role, and should be significantly better in those cases where ill-conditioning affects convergence.

The proposed method can be used with coupled or decoupled formulations. The only requirement for achieving its potential efficiency is skillful programming of sparsity operations. There are no discernible disadvantages.

\section{ACKNOWLEDGEMENTS}

One of the authors (Alvarado) wishes to acknowledge partial NSF support under contract ECS-8822654.

\section{REFERENCES}

1. O. Alsaç, B. Stott and F. L. Alvarado, "Analytical and Computational Improvements in Performance Index Ranking Algorithms," Int. J. of Elect. Power and Energy Syst., Vol. 7, pp. 154-166, July 1985.

2. F. L. Alvarado, "The Sparse Matrix Manipulation System," SIAM Symposium on Sparse Matrices, Gleneden Beach, OR, May 22-24 1989.

3. F. L. Alvarado and M. K. Enns, "Blocked Sparse Matrices in Electric Power Systems," IEEE/PES Summer Meeting, Portland, OR, July 18-23 1976.

4. F. L. Alvarado, D. Yu and R. Betancourt, "Partitioned Sparse $A^{-1}$ Methods," IEEE/PES Summer Meeting, Long Beach, CA July 9-14, 1989.

5. F. C. Aschmoneit, N. M. Peterson and E. Adrian, "State Estimation with Equality Constraints," Proc. of PICA Conf., pp. 427-430, Toronto, May 1977.

6. Å. Björck and I. S. Duff, "A direct method for the solution of sparse linear least squares problems," Linear Alg. and its Applic, Vol. 34, pp. 43-67, 1980.

7. K. A. Clements, G. W. Woodzell and R. C. Burchett, "A New Method for Solving Equality-Constrained Power System Static-State Estimation," IEEE/PES Summer Meeting, July 9-14 1989.

8. I. S. Duff, A. M. Erisman and J. K. Reid, "Direct Methods for Sparse Matrices," Oxford Science Publications, Clarendon Press, 1986.

9. I. S. Duff and J. K. Reid, "A comparison of some methods for the solution of overdetermined systems 
of linear equations," J. Inst. Maths. Applics., Vol. 17 , pp. 267-280, 1976.

10. I. S. Duff and J. K. Reid, "Multifrontal Solution of Indefinite Sparse Symmetric Linear Systems," $A C M$ Trans on Math Software, Vol. 9, Sept. 1983.

11. I. S. Duff, J. K. Reid, N. Munskgaard and H. B. Nielsen, "Direct Solution of Sets of Linear Equations whose matrix is sparse, symmetric and indefinity," $J$. Inst. Maths. Applics., Vol. 23, pp. 235-250, 1979.

12. A. Gjelsvik, S. Aam and L. Holten, "Hachtel's augmented matrix method - a rapid method improving numerical stability in power system static state estimation," IEEE Trans. on Power App. and Syst, Vol. PAS-104, pp. 2987-2993, Nov. 1985.

13. G. Peters and J. H. Wilkinson, "The least squares problem and pseudo-inverse," The Computer Journal, Vol. 13, No. 4, pp. 1534-1542, 1970.

14. F. F. Wu, W. E. Liu and S. Lun, "Observability Analysis and Bad Data Processing for State Estimation with Equality Constraints," IEEE Trans. on Power Syst, Vol. 3, pp. 541-548, May 1988.

15. G. D. Hachtel, "The Sparse Tableau Approach to Finite Element Assembly," Sparse Matrix Computations, pp. 349-363, 1976.

16. J. W. Gu, K. A. Clements, G. R. Krumpholz and P. W. Davis, "The solution of ill-conditioned power system state estimation problems via the method of Peters and Wilkinson," IEEE Trans. on Power App. and Syst, Vol. PAS-102, pp. 3473-3480, Oct. 1983.

17. W. F. Tinney and J. W. Walker, "Direct Solution of Sparse Network Equations by Optimally Ordered Triangular Factorization," Proc. IEEE, Vol. 55, pp. 1801-1809, Nov. 1967.

18. R. Betancourt, "An Efficient Heuristic Ordering Algorithm for Partial Matrix Refactorization," IEEE Trans. on Power Syst, pp. 1181-1187, Aug. 1988.

19. A. Simoes-Costa and V. H. Quintana, "A Robust Numerical Technique for Power System State Estimation," IEEE Trans. on Power App. and Syst, Vol. PAS-100, pp. 691-698, Feb. 1981.

20. A. Simoes-Costa and V. H. Quintana, "An Orthogonal Row Processing Algorithm for Power System Sequential State Estimation," IEEE Trans. on Power App. and Syst, Vol. PAS-100, pp. 37913800, Aug. 1981.

21. W-H. Liu, F. F. Wu, L. Holten, A. Gjelsvik and S. Aam, "Computational Issues in the Hachtel's Augmented Matrix Method for Power System State Estimation," 1987 PSCC, Lisbon, Portugal.

22. F. L. Alvarado and W. F. Tinney, "The Blocked Structure of the Augmented Power System State Estimation Matrix," PSCC Graz, Austria, Aug 1990.

23. G. H. Golub and C. F. VanLoan, "Matrix Computations," $2^{\text {nd }}$ ed, Johns Hopkins Press, 1989.
Fernando L. Alvarado was born in Lima, Peru. He received the BEE and PE degrees from the National University of Engineering in Lima, Peru, the MS degree from Clarkson University, and the Ph.D. degree from the University of Michigan in 1972 . He joined the University of Toledo in 1972. Since 1975 he has been with the University of Wisconsin in Madison, where he is a Professor of Electrical and Computer Engineering.

William F. Tinney received his B. S. and M. S. degrees from Stanford University in 1948 and 1949. He worked for the Bonneville Power Administration from 1950 until his retirement in 1979, at which time he was head of System Analysis. Most of his work has been concerned with power system computer applications and he is presently a consultant in the field. 


\section{DISCUSSION}

I. RADU (Consolidated Edison, New York, NY) and A.) The authors are to be congratulated on an interesting and well written paper. While the basic algorithms and techniques used were known, they have been combined in a novel way and resulted in an improved solution for both internal and, especially external state estimation. For example, the idea of using a blocked sparse matrix formulation for the N/EC method was suggested in a discussion to the OPF paper [8]. The authors provide a equations which is important for the observable solution where the pairing is not trivial (as is the case of external solution). Also, while the state estimator based external solution was introduced in [6], the handling of all injections (except zero equality constraints) in a "Hachtel s manner" improves suited (than a weighting technique) to the implementation of various power flow controls and adjustments.

The authors report that the N/EC method worked remarkably well for the observable solution in several control center implementations. Recently this method was also implemented in the Consolidated Edison EMS as a replacement to the original, vendor supplied, normal equations based program. Note that, due to long line (i.e transformer) connections having low local measurement redundancy and the large number of passive buses, this network can be a litmus test for any state Estimator technique. The N/EC method was implemented with on the fly ordering-factorization (delayed elimination scheme), the factorization in double precision and scaling of the weighting factors. The method is robust. However, it has to be mentioned that, there are only a few measured injections in this system since flow measured subtransmission transformers were explicitly modelled.

With respect to the fact that the authors method is "linked to a topological observability routine" and "relies exclusively on positive-definite matrix techniques" we would like to comment that, perhaps, for the sacrificed. It is well some flexibility is Estimator is the one network application in an EMS which should be very reliable. If it does fail, other Security functions are endangered and more important, its "trustworthiness" in the eyes of the system operator can suffer since he/she can always compare the estimated quantities with the more familiar SCADA measurements. However, in real-time systems, "pathological" cases tend to appear from time to time. Let us consider the following situation: a state estimation run converged in, say, 10 iterations showing some abnormal voltages / flows not flagged as bad data. The typical number of iterations for convergence under similar conditions is 4 or 5 (rather than 10). The question is: are the abnormal values and the unexpected number of iterations due to, say, an emergency condition that should be handled by some rescheduling function or, instead, the abnormal conditions are. simply due to some hidden numerical problems (say small pivots were generated during factorization leading to inaccurate triangular factors). Or, put in a different way: we may want to know if the problem we are dealing with is really solvable with the resources we have at our disposal (computer + algorithm). And, if this is not the case, what should be done to fix the possible, causes of instability. preferably possible causes of instability be done on the fiy during the factorization of the gain matrices.

over the last three decades the general tendency in power system computer analysis has been to do some kind of matrix prearranging (structural or topological analysis) that would permit the factorization process of the problem matrices to be carried out without worrying about numerical problems (pivot observability analysis should, ideally, lead to full rank state estimation matrices, which, hopefully, will go through the numerical factorization process without any problem. When the standard normal equations approach is used, the part of the system defined as observable by the topological observability algorithm usually leads to a positive definite gain matrix. Usually, but not always: that will depend on how the gain matrix is will depend on how the gain matrix is important role here). When it happens that the gain matrix is indeed positive definite (in the numerical sense) it may be factorized without numerical pivoting. As for the methods relying on indefinite matrices (such as N/EC and Hachtel's), blocking schemes as the ones suggested by this and the related paper [A] may permit a positive definite like factorization of the associated indefinite matrices. This is not always guaranteed, several different implementations.

Consider, for example, a radial string of two flow measured branches connected in the reference bus, one with very low impedance (see example in [B] ). Also, while it is true, as the authors state, that critical measurements could be identified by topological observability, numerical checks might provide additional measurement

Numerical problems do occur, qualitative methods sure may help, but are not guaranteed to work in all cases. This being the case, we suggest that, since usually such problems can be traced to a few "bad actors", removing them and thus making a small part of the network temporarily unobservable, would greatly contribute to the reliability of the stat observability algorithm could be used when numerical difficulties appear during the factorization process.

For a discussion on a closely related problem, see [C] which deals with numerical problems associated to the Newton OPF approach.

\section{REFERENCES}

[A] F. I. Alvarado and W. F. Tinney, "State Estimation Using Augmented Blocked Matrices", Paper 90WM 241-0 PWRS, IEEE/PES 1990 Winter Meeting, Atlanta, GA.

[B] A. Monticelli, C. A. F. Murari, and F. F. Wu, "A Hybrid State Estimator: Solving Normal Trans. PAS, vol.PAS-104, No.12, p.3460-3468, December 1985.

[C] A. Monticelli and W-H. E. Liu, "Adaptive Movement Penalty Method for the Newton Optimal Power Flow", Paper 90WM 252-9 PWRS, IEEE/PES 1990 winter Meeting, Atlanta, GA. 
KEVIN A. CLEMENTS (Worcester Polytechnic Institute, Worcester, MA) I congratulate the authors on a most interesting paper describing a new algorithm for solving the equality-constrained power system state estimation problem. The indefinite system of equations that arises in constrained state estimation problems has a very special structure and the algorithm presented by the authors exploits this structure quite nicely.

One issue that was not fully addressed in the paper is that of selecting the pivot elements. This cannot be done by a purely symbolic method since it is possible that candidate pivot blocks may have zero determinants. This problem did not arise in the example presented in the paper but is likely to occur in less well measured systems.

It seems that candidate pivot blocks could be chosen using the minimum degree algorithm but that it is necessary to test the numerical value of the determinant of the block before choosing it as a pivot. Do the authors include such a numerical test in their algorithm?

Manuscript received March 5, 1990.

ANDERS GJELSVIK and SVERRE AAM (Norwegian Electric Power Research Institute, Trondheim, Norway) and LARS HOLTEN (ABB Network Control, Västerảs, Sweden). In this paper the authors have modified the Hachtel's augmented matrix method, and by a block matrix triangularization scheme they avoid the difficulties that arise since the augmented gain matrix is not positive definite. We congratulate the authors for this achievement. What seems to be similar ideas, have appeared simultaneously in [A] .

We would like to make the following comments: In our own work with Hachtel's method, the Harwell MA2? routine was used. It performs numerical tests during factorization and uses $2 \times 2$ pivots when it is found necessary. For this a dynamic data structure is needed. With the present method one seems to be able to use a fixed data structure, determined from sparsity alone. This is a big advantage in a computer implementation. One then has to assume that singularities will not occur. We would like to know whether the authors have encountered problems where the pivot diagonal block matrices have become close to singular during factorization.

The formula (6) for the residual covariance is the one for the unconstrained case. For the constrained case a slightly different formulation has to be used (ref. [14] of paper). In [B] (Section IV and Appendix B) it is described how to obtain residual covariances from Hachtel's augmented matrix in a very efficient way. With slight modifications a similar approach should be useful also with the present formulation.

Table 2 in the paper shows significantly lower condition numbers for the proposed method than for the augmented matrix method. We wonder whether this can be due to the particular system data used. The difference in the two approaches lies in the elimination of the upper left hand portion containing branch measurements in the augmented matrix (1). This operation leads to the $\hat{h}$ elements in (4). One might think that this elimination would in general be more likely to increase the condition number of the matrix than to reduce it, since it is similar to the formation of the normal equations. This would depend on the conditioning of the eliminated part of $H$. In general there may be sources of ill-conditioning also in this part, e.g. mixture of short and long lines. Taking the square root of the "traditional" condition numbers in Table 2 indicates that the systems dealt with are well conditioned. We would appreciate the authors " comments on the observed reduction in condition numbers.

\section{REFERENCES}

[A] R.R. Nucera, Michel L, Gilles, "A blocked sparse matrix formulation for the solution of equality- constrained state estimation", paper 90 WM 234-5 PWRS, IEEE/PES winter Meeting, Atlanta, GA,

Feb. $4-9,1990$

[B] F.F. Wu, W-H. E. Liu, L. Holten, A. Gjelsvik, s. Aam, "Observability analysis and bad data processing for state estimation using Hachtel's augmented matrix method", IEEE Trans. on Power Systems, vol. 3, pp. 604-611, 1988.

Manuscript received February 27, 1990.

P. A. Machado, G. P. Azevedo (CEPEL, Rio de Janeiro/RJ, Brazil): The authors are to be commended for their effort to introduce a method to improve robustness of the state estimation problem.

Our comments regarding the paper are the following: before the factorization process the method establishes blocks corresponding to zero injection measurements. It may happen that an early arrangement of the blocks may lead to an unsolvable system (zero pivot), even when the system has a solution (if the Tinney II criterion of ordering is respected). Let us consider the following example. This network is observable with respect to the $Q-V$ criterion (decoupled version of state estimation)

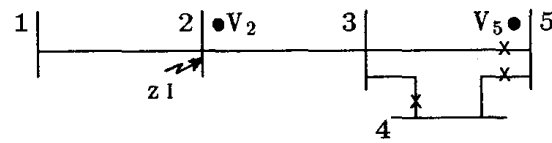

where:

zl - zero injection equality constraint

- - voltage measurement

$\mathrm{x}$ - reactive power flow measurement

After voltage and flow measurements elimination, as suggested in the paper, we obtain the following matrix:

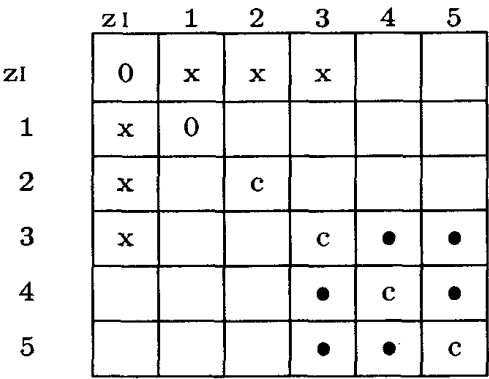

where:

$x$ - original elements

- - fill-in elements

$c$ - diagonal contributions

Using the blocked arrangement we get:

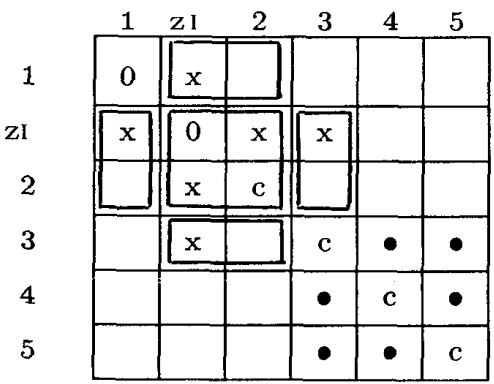


According to the Tinney II criterion the next pivot will be row 1 (one off-diagonal element) which has zero diagonal.

We would appreciate the authors' comments on how the proposed approach can handle situations as the one discussed above.

F. ALVARADO and W. F. TINNEY: We thank the discussers for their excellent discussions. They add important ideas and point out a significant omission in our paper. The main concern in all four discussions is that a sparsityoriented ordering/blocking scheme based strictly on topological properties is vulnerable to numerical breakdown under certain conditions even though the network is topologically observable. The discussion of Machado and Azevedo illustrates this problem quite well. Two of the discussions refer to a concurrent paper by Nucera and Gilles describing the same general blocking scheme which also discusses this condition. Unless the given scheme is modified in some way when this condition exists, it will cause a diagonal block to be singular and thereby cause numerical breakdown. Suggestions and insights provided by the discussers are included in our response to this concern.

There are two aspects to coping with a condition that can cause a diagonal block to be singular: identification of the condition and modification of the ordering/blocking scheme to prevent numerical breakdown from the condition. Identification can be accomplished in either of two basic ways: (1) topologically or (2) numerically. A combination of both is a third possibility. Modifications to cope with an identified singular block can be accomplished in at least three different ways: witholding the block from the sparsity-directed ordering until is has acquired a fill-in from an adjacent elimination, (b) expansion of the block to include another node, or (c) introduction (and subsequent removal) of an innocuous pseudo-measurement into the block.

Identification of conditions requiring some modification of the ordering/blocking scheme can be performed prior to or concurrently with factorization. No matter what precautions are taken prior to factorization, they may not be sufficient to overcome all possible numerical difficulties. This was pointed out by Monticelli and Radu. A network may be observable topologically and unobservable numerically. As a safeguard against this possibility, a test to detect near singularity of diagonal blocks must be performed during factorization no matter what is done beforehand. The identification of singularity concurrently with the recognition of blocks has the advantage of not only detecting this condition, but also the ability to correct it. The most general version of this approach is that taken by the Harwell MA28 routine. In this approach all blocking is done strictly on numerical grounds. For some applications this may be the preferred approach. However, for many other applications we think that detection of the singularity followed by introduction of a pseudo-measurement by compensation may be a better alternative.

Another alternative to prevent singularities on observable systems is described in the Nucera and Gilles paper. In this paper only pairings are permitted (no larger blocks). The observability tree approach of reference [9] in the Nucera and Gilles paper is used to determine when pairings are to occur. That is, a bus may have to be paired with a remote measurement one bus away to resolve its singularity.

Any modification of the sparsity-directed ordering (option (a) above) or block sizes (option (b) above) of the ordering scheme is likely to increase the fill-ins in the matrix factors and thereby increase the computational burden. The increase is small unless there are many conditions that require such modification. Pseudo-measurements introduced to prevent singularity (option (c)) does not cause fill-ins. However, their effect on the estimate must subsequently be nullified by compensation $[A, B]$ in the same manner as identified bad measurements are nullified, and this adds to the computational burden. The burden is small, unless there are many such pseudo-measurements.

The difficulty with singular diagonal blocks is not necessarily disposed of even after a satisfactory initial factorization. If the set of measurements is changed during the estimation process, the condition can occur and must be guarded against. Removal of bad measurements, except by compensation, can create singular diagonal blocks the same as a missing original measurement. Identification and/or modification of operations for critical conditions must therefore be applied again. The tradeoffs may lead to different choices for these operations in the initial and any following factorizations. If compensation (which we recommend) is used to effect the removal of bad measurements, new singularities cannot be introduced and the initial blocking/ordering need not be changed. Compensation can be performed with high efficiency, particularly if approximations are permitted.

Combinations of alternatives (1) and (2) with options (a), (b) or (c) and certain hybrid combinations lead to a variety of modification schemes for overcoming the difficulty. The choice depends to a considerable extent on the schemes used for observability and bad data analysis. Whether the formulation is coupled or decoupled may also affect the choice.

Gjelvik, Aam and Holten express a concern about numerical difficulties caused by the reduction of the line flows. It is possible to come up with a system where widely differing values in the line lengths could lead to a less than ideal condition number. However, the "squaring" of the condition number observed in the normal equations method does not occur. The ill-conditioning, if any, is not artificially added by the numerical method of solution (as it is in the normal equations method), but is inherent in the problem. We have experimented with a wide range of values for line impedances, with the result that the condition numbers deteriorates somewhat for all methods, but the relative merit of the proposed approach remains. Another suggested technique for improving numerical conditioning with or without blocking is the direct evaluation of the algebraic expressions for branch flows in the gain matrix, as suggested by Monticelli and Radu. We were aware of this technique but did not program it and test it. We are not certain whether true or only apparent benefits are derived from this approach.

Gjelvic, Aam and Holten also bring up the subject of covariance matrix computation. In our opinion, only selected elements of the co-variance matrix $R$ are actually required. These elements can be computed "on the fly" as the need for them arises in identifying and compensating for the removal of bad measurements. This computation can be done by using sparse vector methods to find selected residual covariance elements. Typically, this is only a fraction of the effort required to compute a sparse inverse. The paper by $W u$ et al. mentioned by these discussers gives a formula for the computation of the covariance matrix. The following version of the formula is more suitable to the use of blocked matrix methods:

$$
\left[\begin{array}{cccc}
-D & 0 & 0 & H \\
0 & 0 & 0 & G \\
0 & 0 & D & H \\
H^{t} & G^{t} & H^{t} & 0
\end{array}\right]\left[\begin{array}{c}
e_{i} \\
\lambda \\
\mu \\
x
\end{array}\right]=\left[\begin{array}{c}
R *_{i} \\
g \\
z \\
0
\end{array}\right]
$$

In this formula, the upper left hand side has a known left hand side and an unknown right hand side. Selected 
elements of $R$ are computed by selective solutions and partial matrix-vector products.

To summarize, we think that for most problems and under most circumstances a compensation-based modification of our topological approach to blocking is sufficient to eliminate numerical solution concerns, but we recognize that many variants are possible.

[A] L. Mill and T. Van Cutsem, "Implementation of the
Hypothesis Testing Identification in Power System State Estimation," IEEE Transaction on Power Systems, Vol. 3, No. 3, pp. 887-983, August 1988.

[B] I. W. Slutsker, "Bad Data Identification in Power System State Estimation Based on Measurement Compensation and Linear Residual Calculation," IEEE Transaction on Power Systems, Vol. 4, No. 1, pp. 5360, February 1989.

Manuscript received April 9, 1990. 